\title{
Unilateral pedicle screw fixation with transforaminal interbody fusion (UNILIF): 52-patient prospective series
}

\begin{abstract}
Introduction: The success of lumbar spine fusion depends on good patient selection. Bilateral pedicle instrumentation whit transforaminal interbody fusion, now popular, improves fusion rates, and eliminates the necessity for postoperative braces, also allows early mobilization. However, the stress shielding caused by rigid internal fixation is thought to lead to osteopenia and degeneration of adjacent segments. Theatre times, intra-operative complications and costs are increased when pedicle screw fixation is added. The following report is a 52-patient prospective study, with lumbar degenerative disease treated with unilateral pedicle screw fixation plus transforaminal lumbar interbody fusion (UNILIF). We examined the clinical outcomes, fusion rates and also the complications.
\end{abstract}

Material and methods: Prospectively collected clinical and radiological data on patients with degenerative lumbar disease managed by UNILIF. From January 2014 to January 2018, we prospectively collected clinical and radiological data on patients with degenerative lumbar disease managed by UNILIF. Preoperatively and at 2 years, we recorded ODI and VAS. Interbody fusion was analyzed on radiography and on a CT scan.

Results: Mean operation time was $93.5 \mathrm{~min} \pm 16.8$, mean blood loss was $130.8 \mathrm{ml} \pm 210.9$. ODI and VAS were significantly improved $(\mathrm{p}<0.005)$. The fusion rate was $96.8 \%$ on radiographic analysis and was $95.9 \%$ on CT scan analysis. A loosening of the screw was reported, without pain. He was treated with rest and physical therapy, with good evolution. No infections were reported.

Conclusion: Although it is a small group, but with a long follow-up, the UNILIF technique has been shown to be effective and safe for patients with lumbar degenerative disease.

Keywords: Unilateral pedicle instrumentation, interbody fusion, lumbar disease, spondylolysthesis, disck hernation
Volume II Issue 3 - 2019

\author{
Mario Cahueque, ' Juan Armas, ${ }^{2}$ William \\ Lopez, ${ }^{3}$ Enrique Azmitia ${ }^{3,4}$ \\ 'Orthopedic surgeon and spine surgeon, Spine Center, \\ Guatemala \\ ${ }^{2}$ Medical Research, Spine Center, Guatemala \\ ${ }^{3}$ Neurosurgeon and spine surgeon, Spine Center, Guatemala \\ ${ }^{4}$ Head department of Neurosurgery, Hospital Herrera Llerandi, \\ Guatemala
}

Correspondence: Mario Cahueque, Orthopedic Surgeon and Spine Surgeon, Spine Center, Edificio Las Brisas 4to Nivel. Oficina 404A y 404B, 6av. 7-39 zona 10, Guatemala,

Email mariocahueque@gmail.com

Received: April 23, 2019 | Published: May 23, 2019

\section{Introduction}

Transforaminal lumbar interbody fusion (TLIF) was originally labelled by Harms and Rolinger in 1982. ${ }^{1}$ It has turn out to be one of the ordinary standard techniques for the decompression of the ipsilateral foramen and an appropriate interbody fusion. This technique allows the anatomical advantage of not requiring a great retraction of the thecal sac and its contents. High fusion rates have been reported. This procedure is frequently accompanied by the placement of bilateral transpedicular screws in the corresponding segment; this results in immediate rigid segment stabilization that will last while fusion takes places. ${ }^{2,3}$ Some authors have proven that excessive stiffness of such a construct can jeopardize the fusion process due to graft resorption that is in hand due to the lack of stress against end plates. ${ }^{46}$ Scientific evidence in the literature has established that unilateral transpedicular screw fixation, right after fusion, produces radiological results comparable with bilateral fixation: this is done at a lower cost because less amount of implants is used..$^{7-11}$ Minimally invasive transforaminal lumbar interbody fusion (MI-TLIF) was originally described by Foley. ${ }^{12}$

Which is why the absolute requirement for contralateral pedicle fixation to MIS-TLIF is, therefore, controversial. Unilateral pedicle screw fixation as a complement to a MIS-TLIF (technique called UNILIF) has been described since 2006. ${ }^{13}$ Theoretical advantages of this unilateral paramedian posterior approach include a decrease of blood loss, faster surgery procedure, reduced radiation dose, and of course the preservation of the contralateral posterior articular process. It also has similar radiographic fusion rates than open TLIF and a predisposition to yield better clinical results in the immediate postoperative period. ${ }^{7,8,11,14,15}$ We pursued to evaluate the clinical effectiveness of unilateral pedicle screw fixation plus transforaminal lumbar interbody fusion (UNILIF) in our patients with degenerative lumbar disease. The main outcome measure was obtained with Oswestry Disability Index (ODI) and VAS lumbar and VAS Lega pain at 2 year.

\section{Methods}

From January, 2014 to January, 2018, we prospectively collected clinical and radiological data on patients with degenerative lumbar disease managed by a one-level UNILIF, in a private institution by two senior spine surgeons. Each patient signed a consent form. A prospective case-series study was performed in 52 patients with degenerative lumbar disease.

The inclusion criteria were patients older than 18 years and treated by a one-level lumbar arthrodesis for lumbar degenerative diseases (patients with low-grade spondylolisthesis, recurrent disc herniation, extraforaminal disc herniation, and lumbar stenosis with unilateral radiculopathy. Patients with major central stenosis or bilateral radiculopathy, with previous spine surgery with instrumentation, 
tumors, bilateral radicular pain, obese (BMI> 30), osteoporosis were exempt from the study. Of note, patients with unilateral symptomatic radiculopathy but with evidence of radiographic bilateral foraminal stenosis were included in the study and only the symptomatic side was directly decompressed. One-hundred percent of follow-up was achieved. Preoperatively and at 3 years, we documented ODI and VAS lumbar and VAS leg pain. Interbody fusion was analyzed on radiography and on a $\mathrm{CT}$ scan.

\section{Surgical technique}

With the patient in prone position under general anesthesia, use of intensified fluoroscopy images with C-arm, surgical approach was executed on the most symptomatic side. Incision of approx. $3 \mathrm{~cm}$ was performed, using microdiscectomy separators. It is started by placing transpedicular screws of the affected segment and a TLIF separator is used, subsequently a lamina and articular facet osteotomy and medial facetectomy was completed. The Kambi's triangle is located, the medial and lateral root is retracted, preparation was performed and bone graft was delivered in the lumbar intersomatic space. A rectangular bullet-nose cage was implanted. To conclude, unilateral transpedicular screws were placed on the rod.

\section{Outcomes}

Clinical outcomes were evaluated using the ODI, visual analogue scale (VAS) for leg and back pain, patients must complete a minimum of 2 years of follow-up. ODI and VAS were analyzed at 3, 6, 12, 24 and 36 months

\section{Statistical analysis}

Variables were confirmed for normality. Parametric variables were stated as mean (standard deviation), nonparametric as median (interquartile range). Categorical variables were termed as absolute and relative frequencies. Assessment before and after was done through the t-student test. Results were considered significant at a $\mathrm{p}$ value $<0.05$. The analyses were completed using SPSS statistical software, version 20 (SPSS Inc.) (Figures).

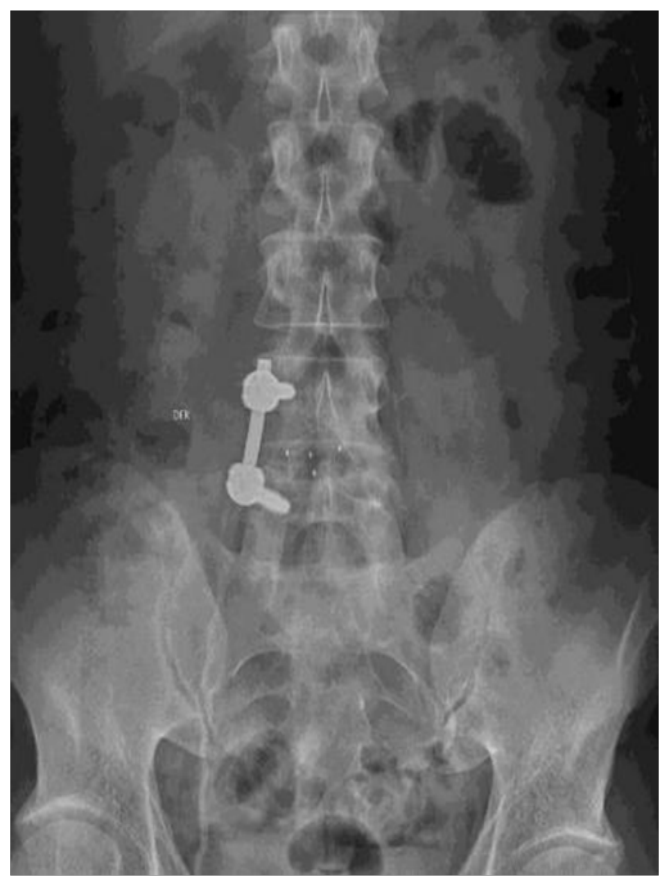

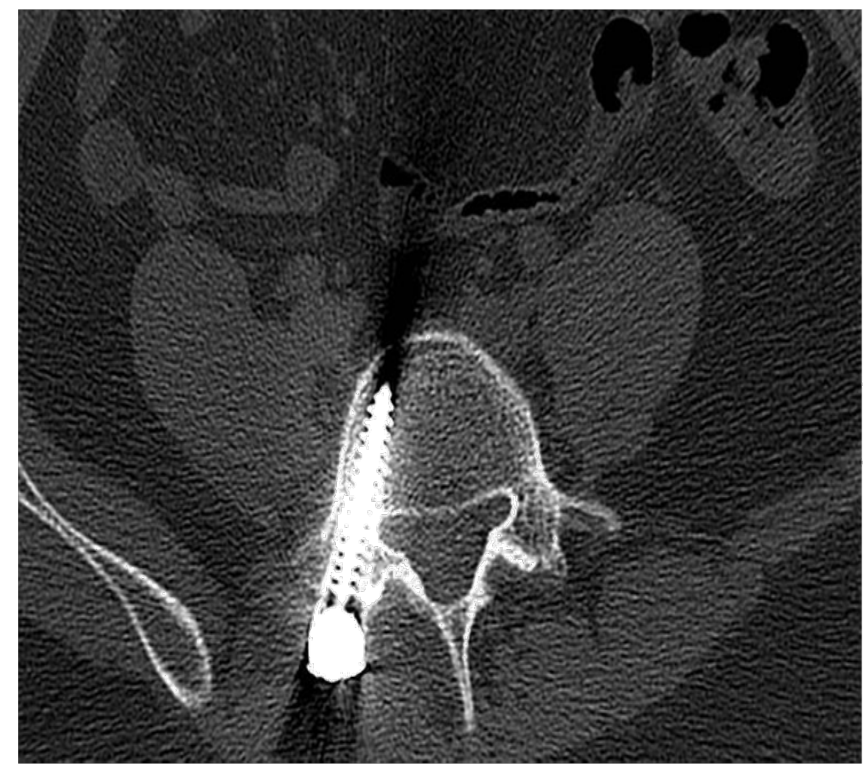
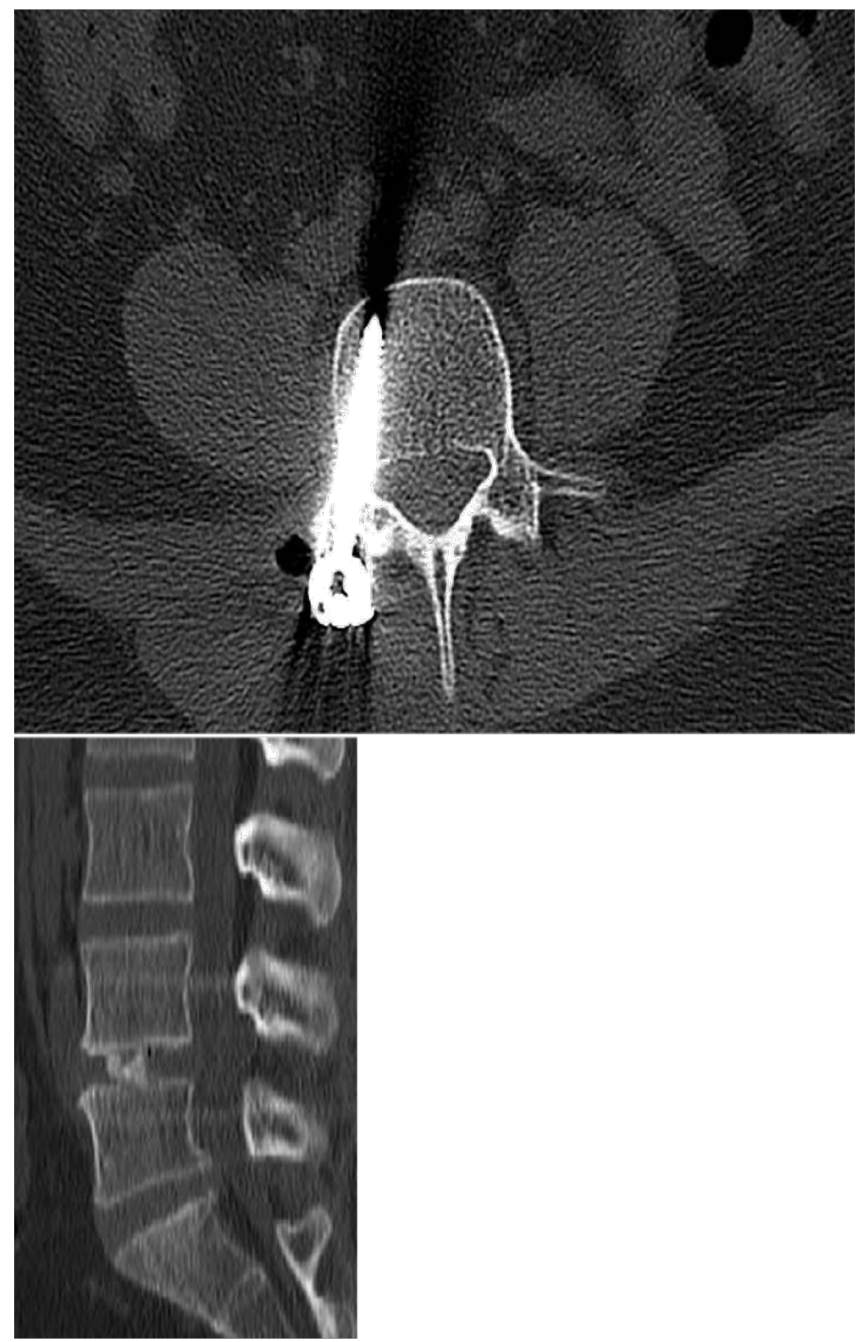


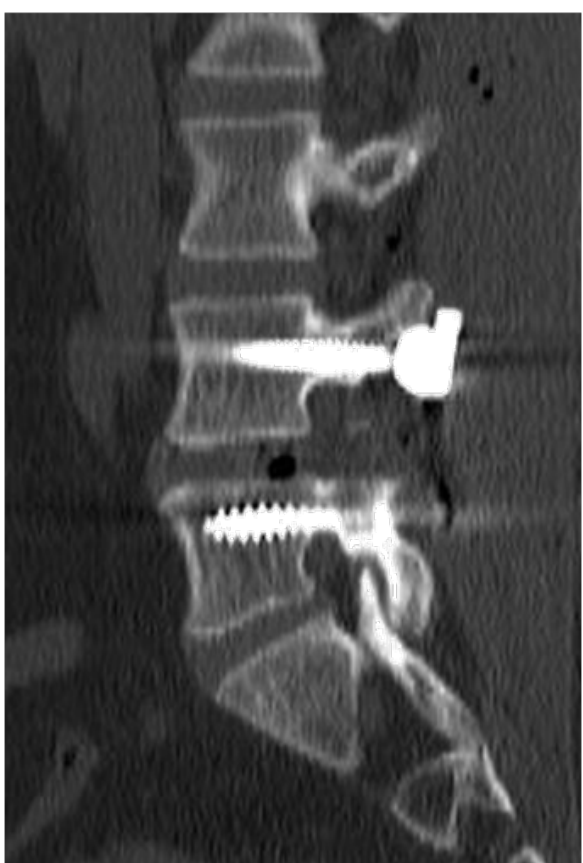

\section{Results}

Baseline characteristics are presented in Table 1. The median age of the patients was 51.1 [29-60] years old. The sample by sex was 15 female patients $(26 \%)$ and 37 male patients $(74 \%)$. The average body mass index was 28 . The most frequent place of radiculopathy was left. Segment most frequently operated was L4 / L5 (85\%), L5 / S1 (12\%), L3 / L4 (3\%). The main surgical indications were, 34 patients presented low-grade spondylolisthesis, with unilateral radiculopathy and 7 patientes with Discopathy/foraminal stenosis. Mean operation time was $93.5 \mathrm{~min} \pm 16.8$, mean blood loss was $130.8 \mathrm{ml} \pm 210.9$.

Table I Demographic data

\begin{tabular}{ll}
\hline & Patients \\
\hline Gender & \\
Female & $15(26)$ \\
Male & $37(74)$ \\
Median Age & $51.1(29-60)^{*}$ \\
Median BMI & 28 \\
Indication & \\
Low-grade spondylilisthesis & $34(65)$ \\
Discopathy/foraminal stenosis & $7(13)$ \\
Extra-foraminal disc herniation & $6(1 \mathrm{I})$ \\
Disc herniation recurrence & $5(9)$ \\
Level of arthrodesis & \\
L3/L4 & $2(3)$ \\
L4/L5 & $44(85)$ \\
L5/SI & $6(12)$ \\
\hline
\end{tabular}

ODI and VAS were significantly enhanced $(p<0.005)$ at the end of the follow-up. There was positive progress from the first 3 months, which was increasing in the subsequent months (Table 2). VAS leg pain improved in all patients. As well as low back pain $(p<0.0001)$. The average hospital time was 3 days, no trans-operative complications, infections or dural tear ware reported. The patients the following day started with walking and immediately physical therapy and rehabilitation. Drainage was routinely placed. No patient needed transfusion. At the 3-year follow-up the fusion rate was $95.1 \%$ on radiographic analysis and was $93.0 \%$ on CT scan analysis. A loosening of the screw was reported, without pain. He was treated with rest and physical therapy, with good evolution. No infections were reported. There was a considerable decrease in the use of analgesics in the first 3 months.

\begin{tabular}{llll}
\hline & ODI (\# patients) & VAS & VAS-Leg Pain \\
\hline Pre-operative & $52.4(52)$ & 6.4 & 7.6 \\
6 months & $23.5(52)$ & 3.8 & 1.7 \\
12 months & $18.7(52)$ & 2.1 & $\mathrm{I} . \mathrm{I}$ \\
24 months & $18.4(36)$ & 1.9 & $\mathrm{I}$ \\
36 months & $\mathrm{I} 5.1(2 \mathrm{I})$ & $\mathrm{I} .2$ & $\mathrm{I}$ \\
& $\mathrm{P}<0.05$ & $\mathrm{P}<0.05$ & $\mathrm{P}<0.05$ \\
\hline
\end{tabular}

\section{Discussion}

TLIF is described to be a regular surgical alternative for patients with isthmic spondylolisthesis and axial pain. Screw fixation go along with this procedure to deliver better stability. Bilateral screw fixation was first introduced but further research has revealed that unilateral screw fixation represents a good choice for preserving stability of the spine. ${ }^{14,16,17}$ In spinal fusion surgery, the need for unilateral or bilateral instrumentation is a controversial matter. Biomechanical studies had demonstrated that unilateral fixation after TLIF delivered less rotational stability and stiffness than bilateral pedicle screw fixation. ${ }^{6,7,17}$ Nevertheless, wide-ranging destruction of tissue structures, which exacerbated considerably the unsteadiness of the spine, was used in these in vitro biomechanical studies. In addition, the maintenance of lumbar stability simply relied on unilateral pedicle screw fixation without any support device. At present, a general consensus was that unilateral instrumentation should be confined to a single-level fusion and not be extended to multilevel fusion because of its inadequate fixation strength.

Kabins et al. ${ }^{8}$ described similar clinical and radiographic fusion results among unilateral instrumentation in compare to the present study. ${ }^{8}$ Suk et al. ${ }^{9}$ designed a prospective study with 87 patients to associate bilateral with unilateral pedicle screw fixation in one or two fused segments, the final conclusion was that unilateral is as effective as bilateral screw fixation in all items appraised: blood loss, operating time, length of hospital stay, clinical outcomes, complication rates and medical expenses. ${ }^{9}$ Recently, Xue compared clinical and radiographic outcomes of patients fixed with unilateral pedicle and bilateral concluding that TLIF with unilateral transpedicular screw fixation is an advantageous treatment choice with better results in terms of operative time, blood loss, and hospital stay for single level disease, matching results were reported by Lin B et al. ${ }^{12}$ It is clear that in invitro models, the rigidity obtained by a bilateral fixation is superior than unilateral. On the other hand, it has been demonstrated that the absence of certain degree of movement can yield to a fusion failure. 
At the 2-year follow-up, our interbody fusion rate measured with CT (93.0) results very similar to those reported by Lin et al. (92.3\%) or in Xiao's meta-analysis of 270 cases $(92.2 \%) .{ }^{17}$ In patients who still did not see a complete fusion, they were asymptomatic, so they did not require a revision surgery.

\section{Limitations}

The current study has several limitations, the most significant is the lack of a control group, the follow-up is long, and it was carried out very well by the same team. The population is small and very heterogeneous, this rule out obese patients and elderly patients, we believe that in this type of patients should be considered biletaral instrumentation.

\section{Conclusion}

This study demonstrates the benefits of minimally invasive onelevel UNILIF in the management of our patients with stable lumbar disease. The results have proven a low rate of perioperative morbidity which is associated with an accelerated worthwhile recovery in the literature. With comparable positive clinical and radiological 2-year outcomes, UNILIF may be a valuable surgical alternative to bi-pedicular screw fixation technique in the management of stable lumbar degenerative diseases. Additional revisions will be required to endorse these results.

\section{Conflicts of interest}

The authors declare that they have no conflict of interest.

\section{References}

1. Harms J, Rolinger H. A one-stager procedure in operative treatment of spondylolistheses: dorsal traction-reposition and anterior fusion (author's transl). Z Orthop Ihre Grenzgeb. 1982;120(3):343-347.

2. Xiao YX, Chen QX, Li FC. Unilateral transforaminal lumbar interbody fusion: a review of the technique, indications and graft materials. $J$ Int Med Res. 2009;37(3):908-917.

3. Harris BM, Hilibrand AS, Savas PE, et al. Transforaminal lumbar interbody fusion: the effect of various instrumentation techniques on the flexibility of the lumbar spine. Spine (Phila Pa 1976). 2004;29(4):E6570.

4. Potter BK, Freedman BA, Verwiebe EG, et al. Transforaminal lumbar interbody fusion: clinical and radiographic results and complications in 100 consecutive patients. J Spinal Disord Tech. 2005;18(4):337-346.
5. McAfee PC, Farey ID, Sutterlin CE, et al. The effect of spinal implant rigidity on vertebral bone density. A canine model. Spine (Phila Pa 1976). 1991;16(6 Suppl):S190-197.

6. McAfee PC, Farey ID, Sutterlin CE, et al. 1989 Volvo Award in basic science. Device-related osteoporosis with spinal instrumentation. Spine (Phila Pa 1976). 1989;14(9):919-926.

7. Goel VK, Lim TH, Gwon J, et al. Effects of rigidity of an internal fixation device. A comprehensive biomechanical investigation. Spine (Phila Pa 1976). 1991;16(3 Suppl):S155-161.

8. Kabins MB, Weinstein JN, Spratt KF, et al. Isolated L4- L5 fusions using the variable screw placement system: unilateral versus bilateral. $J$ Spinal Disord. 1992;5(1):39-49.

9. Suk KS, Lee HM, Kim NH, et al. Unilateral versus bilateral pedicle screw fixation in lumbar spinal fusion. Spine (Phila $\mathrm{Pa}$ 1976). 2000;25(14):1843-1847.

10. Beringer WF, Mobasser JP. Unilateral pedicle screw instrumentation for minimally invasive transforaminal lumbar interbody fusion. Neurosurg Focus. 2006;20:E4.

11. Deutsch H, Musacchio MJ Jr. Minimally invasive transforaminal lumbar interbody fusion with unilateral pedicle screw fixation. Neurosurg Focus. 2006;20(3):E10.

12. Xue H, Tu Y, Cai M. Comparison of unilateral versus bilateral instrumented transforaminal lumbar interbody fusion in degenerative lumbar diseases. Spine J. 2012;12(3):209-215.

13. Foley KT, Holly LT, Schwender JD. Minimally invasive lumbar fusion. Spine. 2003;28(15 Suppl):S26-35.

14. Tuttle J, Shakir A, Choudhri HF. Paramedian approach for transforaminal lumbar interbody fusion with unilateral pedicle screw fixation. Technical note and preliminary report on 47 cases. Neurosurg Focus. 2006;20(3):E5.

15. Cao Y, Chen Z, Jiang C, et al. The combined use of unilateral pedicle screw and contralateral facet joint screw fixation in transforaminal lumbar interbody fusion. Eur Spine J. 2015;24(11):2607-2613.

16. Huang $\mathrm{P}$, Wang Y, Xu J, et al. Minimally invasive unilateral pedicle screws and a translaminar facet screw fixation and interbody fusion for treatment of single-segment lower lumbar vertebral disease: surgical technique and preliminary clinical results. J Orthop Surg Res. 2017;12(1):117.

17. Xiao SW, Jiang H, Yang LJ, et al. Comparison of unilateral versus bilateral pedicle screw fixation with cage fusión in degenerative lumbar diseases: a meta-analysis. Eur Spine J. 2015;24(4):764-774. 